\title{
Female Adnexal Tumor of Wolffian Origin Presenting as Paratubal / Broad Ligament Masses: A Case Report
}

\author{
Sedigheh Ghasemian Dizaj Mehr (iD) 1, ${ }^{*}$, Setareh Akhavan (iD) ${ }^{2}$, Behnaz Jahanbin (iD ${ }^{3}$, Sharzad \\ Sheikhhasani (iD ${ }^{2}$ and Farzaneh Rashidi Fakari (iD ${ }^{4}$ \\ ${ }^{1}$ Department of Obstetrics and Gynecology, School of Medicine, Urmia University of Medical Sciences, Urmia, Iran \\ ${ }^{2}$ Department of Oncologic Gynecology, Vali-Asr Hospital, Tehran University of Medical Sciences, Tehran, Iran \\ ${ }^{3}$ Department of Pathology, Cancer Research Institute, Imam Khomeini Hospital Complex, Tehran University of Medical Sciences, Tehran, Iran \\ ${ }^{4}$ Department of Midwifery, School of Medicine, North Khorasan University of Medical Sciences, Bojnurd, Iran \\ "Corresponding author: Department of Obstetrics and Gynecology, School of Medicine, Urmia University of Medical Sciences, Urmia, Iran. Email: dr.ghassemian@yahoo.com
}

Received 2020 August 08; Revised 2021 June 16; Accepted 2021 June 21.

\begin{abstract}
Introduction: A female adnexal tumor of probable Wolffian origin (FATWO) is a rare type of gynecological tumor that is difficult to diagnose based on laboratory and radiologic studies. Although FATWO is considered a benign tumor, recurrence and metastasis have been reported. Currently, there is no best surgical and surveillance approach for FATWO. We presented two Wolffian tumors as paratubal / broad ligament masses.

Case Presentation: This is a report of a 32-year-old woman with vaginal discharge and incidentally pelvic mass diagnosis who underwent conservative surgery with a frozen section based on the Wolffian duct tumor. Immunohistochemically, the results confirmed FATWO. The patient was free of recurrence 20 months after conservative surgery.

Conclusions: Although FATWO has benign behavior, some types of this tumor have malignant features, and there is no clear recommendation regarding optimal treatment and surveillance of FATWO. Therefore, based on the literature, pathologists are recommended to utilize a wide array of immunohistochemical stainings to offer appropriate diagnosis and optimal surgery to patients. Moreover, patients should be followed up for a long-term period until we have enough knowledge in the field.
\end{abstract}

Keywords: Ligament, Tumor, Wolffian Tumor

\section{Introduction}

A Wolffian tumor is a rare type of tumor that is presumed to originate in mesonephric remnants. It was originally described by Karminejad and Scully in 1973 as FATWO $(1,2)$. Reproductive organs, including the broad ligament, mesosalpinx fallopian tubes, ovaries, and peritoneum originate from the mesonephric system (Wolffian duct) (2).

FATWO occurs more commonly in the broad ligament as a benign lesion, and it should be considered as a lowgrade malignancy. Recurrence and metastasis of FATWO have been reported $(2,3)$.

Because of the rarity of this tumor, there is no recommendation about its management and treatment. We reported a rare case of FATWO in the broad ligament paratubal with infiltration of tumor cells at the seromuscular layer of the fallopian tube.

\section{Case Presentation}

A 32-year-old woman, gravida 4, live 1, and abortion 3 was diagnosed with left ovarian mass and endometrial polyp after evaluation for vaginal discharge by sonography. Her previous gynecological history included a normal vaginal delivery and three spontaneous abortions. In the vaginal examination, there was fullness in the left adnexa, separated from the uterine, mobile, and without tenderness. Transvaginal ultrasound revealed a normal size uterus and echogenic solid mass with dimensions of $14 \times 6$ $\mathrm{mm}$ in the endometrial cavity, which could be due to endometrial polyp and a well-defined highly echogenic solid mass with a diameter of $60 \times 60 \mathrm{~mm}$ adjacent to the left ovary with normal size ovaries (Figure 1). Magnetic resonance imaging (MRI) confirmed this mass. A T1-weighted fat saturation contrast-enhanced MRI showed a $65 \times 65$ $\mathrm{mm}$, well-defined mass with heterogeneous enhancement in the left broad ligament that was attached to the lower 
part of the left ovary (Figure 2). The chest X-ray was unremarkable. Serum tumor markers were Ca125:26 u/mL, CA19-9: $7 \mathrm{u} / \mathrm{mL}$, and CEA: $0.5 \mathrm{ng} / \mathrm{mL}$.

First, to exclude endometrial pathology, the patient underwent hysteroscopy curettage and polyp resection with the histopathologic finding being endometrial polyp with secretory changes. After this evaluation, laparotomy was intraoperatively performed, and two fleshy well-defined solid masse with the size of $10 \times 10 \mathrm{~cm}$ and $2 \times 3 \mathrm{~cm}$ were found in the left paratubal, broad ligament, distinct from the left ovary and near the left fallopian tube (Figures 3 and 4). There were no macroscopic intra-abdominal lesions. Both masses were removed and examined with a frozen section, and Sertoli cell tumors were considered. However, due to the location of the tumor in the broad ligament and the normal ovary, the Wolffian duct tumor was more probable. Therefore, only left salpingectomy and mass resection were performed, and the patient was discharged without any complication. Grossly, this mass was $10 \times 8 \times 4 \mathrm{~cm}$, and cut sections showed a solid homogeneous friable surface with small cysts; however, the small mass was $3 \times 3 \times 2$ cm resembling a large mass. Microscopically, the small mass had sieve-like and tubulocystic appearances with intraluminal bright eosinophilic secretions and necrosis. Mitosis count was 5 per 10 high power fields in hot spot areas. Infiltration of tumor cells existed at the serosal surface and muscular layer of the left fallopian tube near this mass. Immunohistochemically, the tumor cells were positive for calretinin, inhibin, vimentin, CD56, WT1, and cytokeratins (CK) 7 and 19, but negative for epithelial membrane antigen (EMA), CD10, AFP, and Glypican-3. FATWO was confirmed based on this pathologic finding. Twenty months after conservative surgery, evidence of recurrence was not observed during close follow-up by sonography. It should be noted that informed consent was obtained from the patient for this report.

\section{Discussion}

FATWO originates in mesonephric remnants and presents as an expansible homogeneous tumor suspended in the broad ligament (2).

Some adnexal tumors are close to the ovary, and this is the reason why this type of tumor is reported as ovarian masses (4). FATWO is an uncommon tumor presented at different ages with different ranges of size $(5,6)$. Patients with large FATWO may present with abdominal pain, but many patients remain asymptomatic and are diagnosed incidentally $(3,4)$. Microscopically, FATWO exhibits different features from solid with spindled cells to various degrees of tubule formation and cystic structures $(2,3)$. Diagnosing FATWO was supported by the immunohistochemical profile, and staining should be positive for calretinin, $\mathrm{CK} 7, \mathrm{CK} 8, \mathrm{CK} 10$, and vimentin, but negative for EMA, the carcinoembryonic antigen (CEA), and CK20 (2, 7, 8). Molecular alterations in FATWO are still unclear (3). There are no specific serum biomarkers for this tumor. Moreover, there are no specific radiologic findings in the Wolffian duct tumor. A computed tomography scan is more accurate than ultrasound in determining the tumor origin, and it is heterogeneously enhanced in CTS $(3,7)$.

Due to cystic denegation in the Wolffian duct tumor and since it is slightly hyperintense, it is difficult to differentiate between subserosal myoma and the thecomas with FATWO in MRI (9). This tumor has benign behavior, but some recurrent and metastatic cases have been reported (10). It is reported that the size above $10 \mathrm{~cm}$, necrosis, capsular invasion, a high number of mitosis, positive staining for CD117, and overexpression of ki-67 are correlated with malignant behavior $(4,11)$. Therefore, FATWO should be considered as a low-grade malignancy, and the patient should be closely monitored after tumor resection (2). Pathologically, the Wolffian duct tumor has some differential diagnoses, such as systolic Leydig cell tumor, endometriosis carcinoma of the fallopian tube, clear cell carcinoma, and granulosa cell tumor $(2,12,13)$. Immunohistochemistry may help exclude these tumors from FATWO. Negative EMA is an advantage in epithelial neoplasms, and positive CD10 staining is helpful to sex cord-stromal tumors. Moreover, FATWO is extra tubal, whereas FATWOlike tumors are intramural masses $(8,14)$. However, it is difficult to differentiate FATWO from these tumors and we should pay attention to all the characteristics of these tumors. In our patient, due to the lack of androgenic symptoms, location of the tumor on the base of the paratubal, broad ligament, extraluminal infiltration of the fallopian tube, and pathologic and immunohistochemical findings, the Wolffian duct tumor was recommended. We performed conservative surgery due to a frozen section pathology report. The patient was disease-free after a 20month follow-up. Patients who were treated with tumor resection alone have the most recurrent FATWO within two years (15). In the pathology of our patient, there was an infiltration of tumor cells in the sermuscular layer of the left fallopian tube; it is an invasive feature that should be considered by clinicians. FATWO is a low-grade malignancy, 

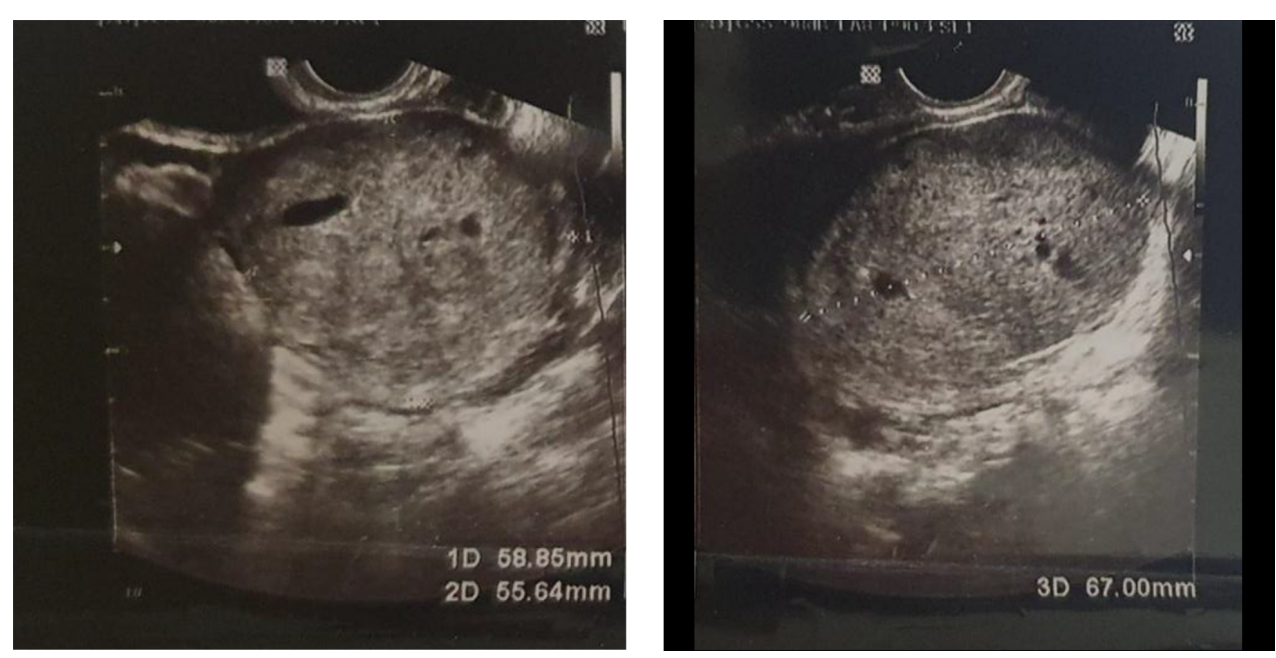

Figure 1. Transvaginal ultrasound as a well-defined highly echogenic solid mass with a diameter of $60 \times 60$ mm containing a few small cystic areas in the left adnexa.
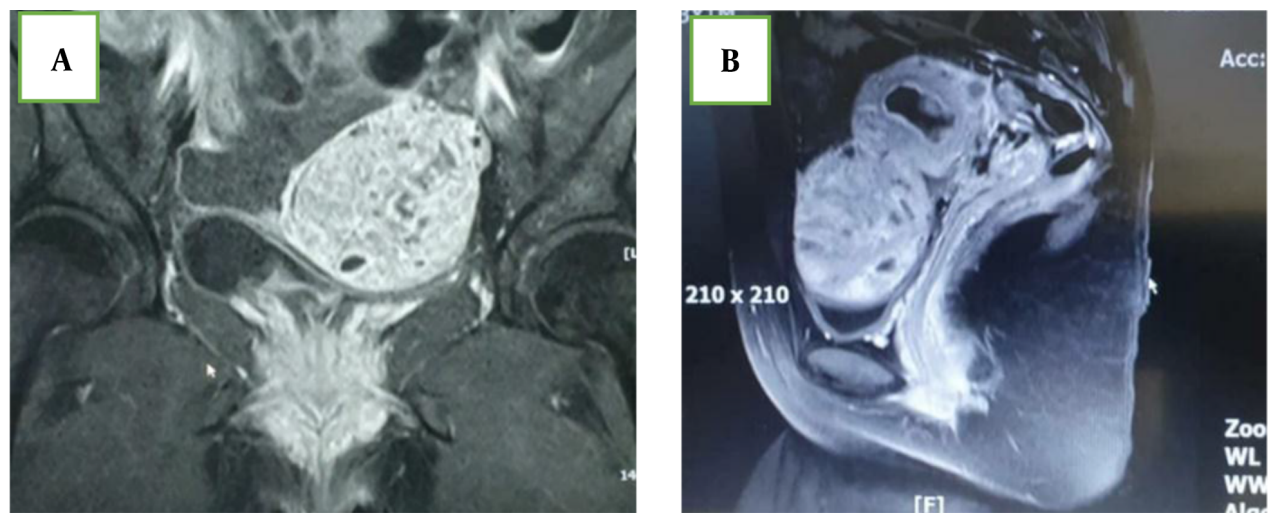

Figure 2. (A) Coronal and (B) Sagittal T1-weighted fat saturation contrast-enhanced magnetic resonance images showing a $65 \times 65$ mm, well-defined mass with heterogeneous enhancement.
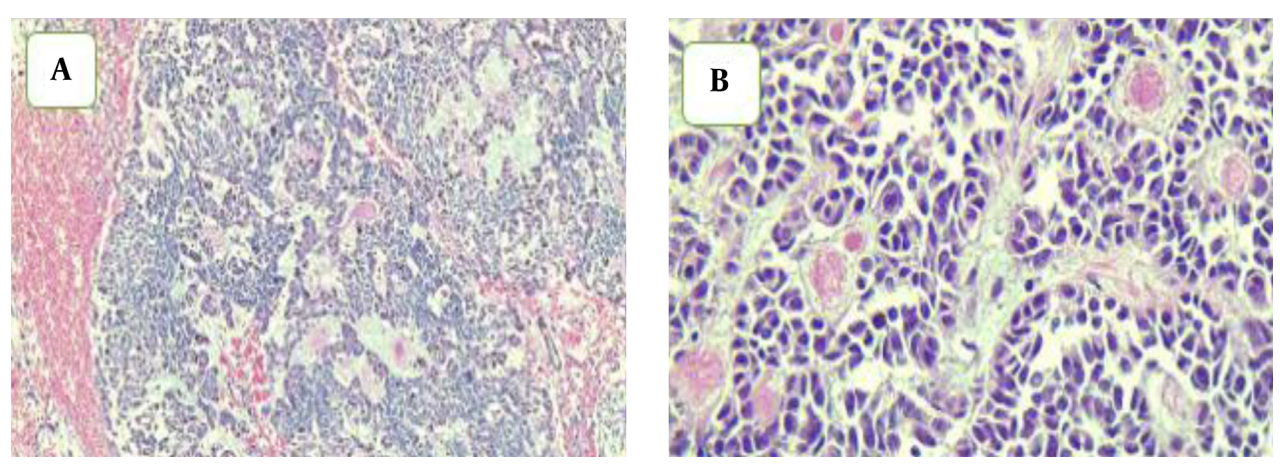

Figure 3. (A) Sieve-like and tubulocystic appearance of the tumor and (B) Intraluminal bright eosinophilic secretions. 


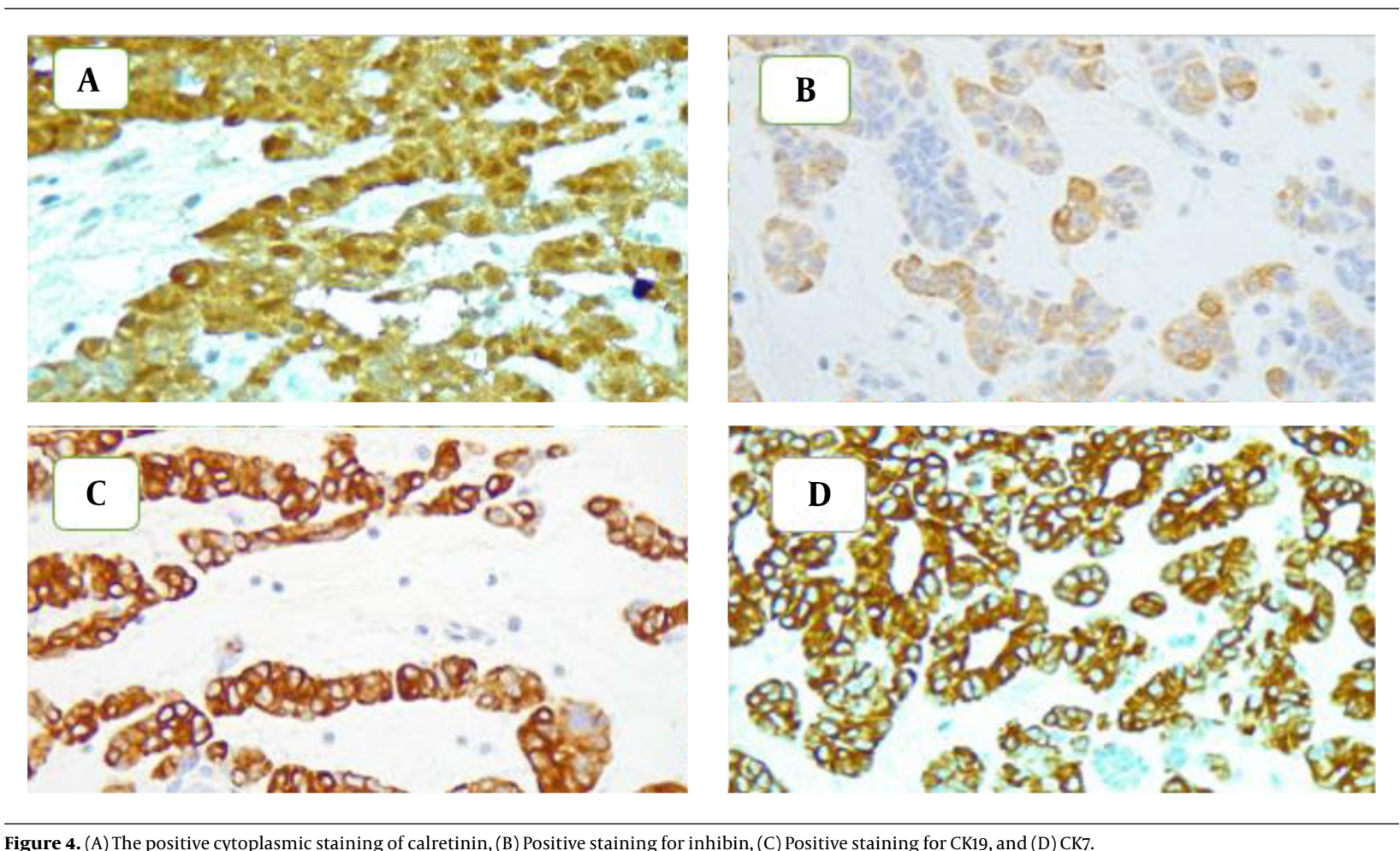

Figure 4. (A) The positive cytoplasmic staining of calretinin, (B) Positive staining for inhibin, (C) Positive staining for CK19, and (D) CK7.

and patients should be closely monitored after its removal (2).

\subsection{Conclusions}

Although FATWO has benign behavior, it may have malignant features, and there is no clear recommendation regarding its optimal treatment and surveillance. Therefore, based on the literature, pathologists are recommended to utilize a wide array of immunohistochemical stainings to help appropriately diagnose the disease. Therefore, optimal surgery is performed, and patients should be followed up for a long-term period until we have enough knowledge in the field.

\section{Acknowledgments}

The authors would like to thank all the members of the Obstetrics and Gynecology Department of the Imam Khomeini Hospital, Tehran, for their help and suggestions.

\section{Footnotes}

Authors' Contribution: S.GH: Project administration, writing, review, and editing, Data Collection; S.A: Project administration, writing, review, and editing, supervision; B.J: Project administration, writing, review, and editing, Data Collection; SH.SH: Project administration, writing, review, and editing, Data Collection; F.R: Project administration, writing, review, and editing, Data Collection.

Conflict of Interests: None declared.

Funding/Support: None declared.

Informed Consent: Informed consent was obtained from the patient.

\section{References}

1. Kariminejad MH, Scully RE. Female adnexal tumor of probable wolffian origin.A Distinctive Pathologic Entity. Cancer.1973;31(3):671-7. doi: 10.1002/1097-0142(197303)31:3<671::Aid-cncr2820310328>3.0.Co;2-k.

2. Crum CP, Lee KR, Nucci MR, Granter SR, Howitt BE, Parast MM. Diagnostic Gynecologic and Obstetric Pathology. Elsevier Health Sciences; 2017.

3. Shalaby A, Shenoy V. Female Adnexal Tumor of Probable Wolffian Origin: A Review. Arch Pathol Lab Med. 2020;144(1):24-8. doi: 10.5858/arpa.2019-0152-RA. [PubMed: 31469585].

4. Liu L, Fang Q, Xing Y. Female adnexal tumor of probable Wolffian origin arising from mesosalpinx: A case report and review. $J \mathrm{Ob}$ stet Gynaecol Res. 2018;44(9):1859-63. doi: 10.1111/jog.13704. [PubMed: 29974617].

5. Zhang W, Valente PT, Riddle ND. Female Adnexal Tumors of Probable Wolffian Origin with a Biphasic Histologic Growth Pattern and Positive for C-kit. Hum Pathol. 2016;4:46-9. doi: 10.1016/j.ehpc.2015.08.006. 
6. Li CC, Qian ZR, Hirokawa M, Sano T, Pan CC, Hsu CY, et al. Expression of adhesion molecules and Ki-67 in female adnexal tumor of probable Wolffian origin (FATWO): report of two cases and review of the literature. APMIS. 2004;112(6):390-8.

7. Cossu A, Casula M, Paliogiannis P, Tanda F, Palomba G, Sini MC, et al. Female Adnexal Tumors of Probable Wolffian Origin (FATWO): A Case Series With Next-Generation Sequencing Mutation Analysis. Int J Gynecol Pathol.2017;36(6):575-81. doi:10.1097/PGP.0000000000000368. [PubMed: 28463911].

8. Fukunaga M, Bisceglia M, Dimitri L. Endometrioid carcinoma of the fallopian tube resembling a female adnexal tumor of probable wolffian origin. Adv Anat Pathol. 2004;11(5):269-72. doi: 10.1097/01.pap.0000138141.88763.6a. [PubMed: 15322493].

9. Matsuki M, Kaji Y, Matsuo M. Female adnexal tumour of probable Wolffian origin: MR findings. Br J Radiol. 1999;72(861):911-3. doi: 10.1259/bjr.72.861.10645202. [PubMed: 10645202].

10. Adiguzel C, Eser EP, Baysal S, Yilmaz ESS, Baser E. Female adnexal tumor of probable wolffian origin, FATWO: Report of a rare case. Int $J$ Gynecol Cancer. 2015.

11. Lesin J, Forko-Ilic J, Plavec A, Planinic P. Management of Wolffian duct tumor recurrence without chemotherapy. Arch Gynecol Obstet. 2009;280(5):855-7. doi: 10.1007/s00404-009-1024-1. [PubMed:
19288122].

12. Mirkovic J, Dong F, Sholl LM, Garcia E, Lindeman N, MacConaill L, et al. Targeted Genomic Profiling of Female Adnexal Tumors of Probable Wolffian Origin (FATWO). Int J Gynecol Pathol. 2019;38(6):543-51. doi: 10.1097/PGP.0000000000000545. [PubMed: 30134342].

13. Tiltman AJ, Allard U. Female adnexal tumours of probable Wolffian origin: an immunohistochemical study comparing tumours, mesonephric remnants and paramesonephric derivatives. Histopathology. 2001;38(3):237-42. doi: 10.1046/j.13652559.2001.01086.x. [PubMed: 11260305].

14. Rosen C, Reardon E, Shyu S, Terhune J, Saats P, Ioffe O, et al. Wolffian tumor (female adnexal tumor of Wolffian origin) presenting as a pelvic side wall mass: Report of a case. SAGE Open Med. 2019;7:2050313X19839534. doi: 10.1177/2050313X19839534. [PubMed: 30967956]. [PubMed Central: PMC6444779].

15. Wakayama A, Matsumoto H, Aoyama H, Saio M, Kumagai A, Ooyama $\mathrm{T}$, et al. Recurrent female adnexal tumor of probable Wolffian origin treated with debulking surgery, imatinib and paclitaxel/carboplatin combination chemotherapy: A case report. Oncol Lett.2017;13(5):34038. doi: 10.3892/ol.2017.5874. [PubMed: 28529572]. [PubMed Central: PMC5431746]. 